\title{
GEVREY WELL POSEDNESS FOR A SECOND ORDER WEAKLY HYPERBOLIC EQUATION WITH NON REGULAR IN TIME COEFFICIENTS
}

\begin{abstract}
By
Alessia AsCANELli

Abstract. We consider a second order weakly hyperbolic equation with coefficients depending both on time and space. We assume the coefficients of the equation to have some kind of Hölder behavior with respect to time, and we add for the coefficients of the lower order terms an appropriate Levi condition. We prove Gevrey well posedness of the Cauchy problem for this equation for a small enough Gevrey index.
\end{abstract}

\section{Introduction}

It is well known that the Cauchy problem for a strictly hyperbolic equation with regular (Lipschitz continuous) coefficients with respect to time is $C^{\infty}$ well posed. But if the equation is weakly hyperbolic or if the coefficients are less than Lipschitz continuous, then $C^{\infty}$ well posedness may fail to be true, as shown by counterexamples in [4], [6], [9]. Anyway, it is always possible to find well posedness in Gevrey classes.

In this paper we are going to study a weakly hyperbolic equation of the second order with coefficients in $t, x$, not regular in time, and we are going to find results of well posedness in Gevrey classes $G^{\sigma}$. We remind that $G^{\sigma}\left(\mathbf{R}^{n}\right)=$ $\bigcup_{A>0} G_{A}^{\sigma}\left(\mathbf{R}^{n}\right)$, where for $\sigma \geq 1, A>0$

$$
G_{A}^{\sigma}\left(\mathbf{R}^{n}\right)=\left\{u(x) ;\|u\|_{\sigma, A}<\infty\right\}, \quad\|u\|_{\sigma, A}=\sup _{x \in \mathbf{R}^{n}, \alpha \in \mathbf{Z}_{+}^{n}}\left|\partial^{\alpha} u(x)\right| A^{-|\alpha|}|\alpha| !^{-\sigma}
$$

Let us consider at first the homogeneous weakly hyperbolic Cauchy problem

Received June 22, 2005. 


$$
\begin{aligned}
& \left\{\begin{array}{l}
u_{t t}-\sum_{i, j=1}^{n} a_{i, j}(t, x) u_{x_{i} x_{j}}=0, \quad t \in[0, T], x \in \mathbf{R}^{n}, \\
u(0, x)=u_{0}(x) \\
u_{t}(0, x)=u_{1}(x),
\end{array}\right. \\
& a(t, x, \xi)=\sum_{i, j=1}^{n} a_{i j}(t, x) \xi_{i} \xi_{j} \geq 0, \quad t \in[0, T], x, \xi \in \mathbf{R}^{n},|\xi|=1 .
\end{aligned}
$$

If $a_{i j}=a_{i j}(t) \in C^{m, k}[0, T], m \in \mathbf{N}, k \in(0,1]$, then problem (1.1) is well posed in $G^{\sigma}$ for

$$
1 \leq \sigma<1+\frac{m+k}{2}
$$

[5]. This result is optimal, as proved in the same paper by producing a counterexample.

If $a_{i j}=a_{i j}(t, x) \in C^{m, k}\left([0, T] ; G^{\sigma}\right)$, then Gevrey well posedness holds with [11]

$$
1 \leq \sigma<\min \left\{1+\frac{m+k}{2}, 2\right\}
$$

Both in [5] and in [11] the non homogeneous equation

$$
u_{t t}-\sum_{i, j=1}^{n} a_{i, j}(t, x) u_{x_{i} x_{j}}+\sum_{j=1}^{n} b_{j}(t, x) u_{x_{j}}+c(t, x)=0
$$

is also considered, respectively with $b_{j}=b_{j}(t), c=c(t)$ and $b_{j}=b_{j}(t, x), c=$ $c(t, x)$. The authors found that the Cauchy problem for equation (1.2) is $G^{\sigma}$-well posed for

$$
1 \leq \sigma<\min \left\{1+\frac{m+k}{2}, 2\right\}
$$

assuming $b_{j} \in L^{1}([0, T]), j=1, \ldots, n$. To overcome the bound $\sigma<2$, Levi conditions on the first order term are necessary, also in the case of regular in time coefficients [3].

Here we consider the Cauchy problem

$$
\left\{\begin{array}{l}
P\left(t, x, D_{t}, D_{x}\right) u(t, x)=0, \quad t \in[0, T], x \in \mathbf{R}^{n}, \\
u(0, x)=u_{0}(x) \\
\partial_{t} u(0, x)=u_{1}(x)
\end{array}\right.
$$


for the second order operator

$$
P=D_{t}^{2}-\alpha(t) Q\left(t, x, D_{x}\right)+b\left(t, x, D_{x}\right)+c(t, x),
$$

where $D=\frac{1}{\sqrt{-1}} \partial$ and

$$
\left\{\begin{array}{l}
Q(t, x, \xi)=\sum_{i, j=1}^{n} q_{i j}(t, x) \xi_{i} \xi_{j} \\
b(t, x, \xi)=\sum_{j=1}^{n} b_{j}(t, x) \xi_{j} .
\end{array}\right.
$$

The condition of weak hyperbolicity for $P$ is expressed here below:

$$
\left\{\begin{array}{l}
\alpha(t) \geq 0, \quad t \in[0, T], \\
Q(t, x, \xi) \geq q_{0}|\xi|^{2}, \quad q_{0}>0, t \in[0, T], x, \xi \in \mathbf{R}^{n} .
\end{array}\right.
$$

As to the regularity of the coefficients of $P$, we assume, as in [5], [11], that

$$
\left\{\begin{array}{l}
\alpha \in C^{N}[0, T]:=C^{m, k}[0, T], \quad N=m+k, m \in \mathbf{N}, k \in(0,1], \\
q_{i, j} \in C^{\chi}\left([0, T] ; G^{\sigma}\right), \quad \chi \in(0,1), i, j=1, \ldots, n, \sigma \geq 1, \\
b_{j}, c \in C\left([0, T] ; G^{\sigma}\right), \quad j=1, \ldots, n .
\end{array}\right.
$$

In Theorem 2.1 we consider the homogeneous operator (1.4), that is (1.4) with $b \equiv c \equiv 0$. We find for problem (1.3) Gevrey well posedness, with different bounds for the Gevrey index $\sigma$ according to the relationship between $N$ and $\chi$. More precisely we prove the following:

i) If $\chi<N /(2+N)$, then the Hölder behavior and the ellipticity of the symbol $Q$ dominate, and we have $G^{\sigma}$ well posedness if

$$
1 \leq \sigma<\frac{1}{1-\chi} .
$$

The bound $1 /(1-\chi)$ is sharp in the strictly hyperbolic case (see the counterexample in [4]), so it clearly cannot be improved for weakly hyperbolic equations.

ii) If $\chi \geq N /(2+N)$, then the $C^{N}$ behavior dominates and we have $G^{\sigma}$ well posedness if

$$
1 \leq \sigma<1+\frac{N}{2} .
$$

Thus, we find the same bound as [5] but here with coefficients depending also on space, and we remove the condition $\sigma<2$ of [11] thanks to the special structure of (1.4). 
In Theorem 3.1 we consider operator (1.4) in its general form, and we look at the right Levi conditions to be put on the term $b$ in order to obtain well posedness of the Cauchy problem (1.3) in Gevrey classes with the same bounds for $\sigma$ as in Theorem 2.1. We suppose so (1.4) to satisfy conditions (1.5), (1.6), and for the lower order terms we assume the following Levi-type condition:

$$
\left|\partial_{x}^{\beta} b_{j}(t, x)\right| \leq C A^{|\beta|} \beta !^{\sigma} \alpha(t)^{\gamma}, \quad(t, x) \in[0, T] \times \mathbf{R}^{n}, \beta \in \mathbf{Z}_{+}^{n},
$$

$j=1, \ldots, n, \gamma \in[0,1 / 2]$ to be chosen. Then we prove the following:

i) $\chi<N /(2+N)$,

$$
\gamma \geq 1-\frac{1}{2 \chi} \Rightarrow G^{\sigma} \text { well posedness, } \quad 1 \leq \sigma<\frac{1}{1-\chi}
$$

ii) $\chi \geq N /(2+N)$,

$$
\gamma \geq \frac{1}{2}-\frac{1}{N} \Rightarrow G^{\sigma} \text { well posedness, } \quad 1 \leq \sigma<1+\frac{N}{2} .
$$

REMARK 1.1. In both cases, the relation between the Levi exponent $\gamma$ and the Gevrey index $\sigma$ is

$$
\gamma>\frac{\sigma-2}{2(\sigma-1)}
$$

Thus we can take:

- $\gamma=0$ if $\sigma<2$, result which is in line with [3], [8]: $G^{\sigma}$ well posedness holds without assuming any Levi condition;

- $\gamma=1 / 2$ if $\sigma \rightarrow \infty$, result which is in line with [5], [10], since

$$
\left|\partial_{x}^{\beta} b(t, x, \xi)\right| \leq C_{\beta} \sqrt{a(t, x, \xi)}, \quad t \in[0, T], x, \xi \in \mathbf{R}^{n}, \beta \in \mathbf{Z}_{+}^{n}
$$

is a sufficient Levi condition to obtain $C^{\infty}$ well posedness in the case of analytic coefficients.

The results in this paper are obtained following this scheme:

1) we regularize the characteristic roots of $P$ (which are not distinct and not regular in time), and then we factorize the operator $P$ by means of the approximated roots;

2) we reduce the equation $P u=f$ to an equivalent first order $2 \times 2$ system $L U=F$ with

$$
L=\partial_{t}+i \Lambda\left(t, x, D_{x}\right)+R\left(t, x, D_{x}\right)
$$


where $\Lambda$ is a real diagonal matrix of symbols of order 1 , and

$$
\left|D_{\xi}^{\alpha} \partial_{x}^{\beta} R(t, x, \xi)\right| \leq c_{\alpha, \beta}(t) A^{|\beta|} \beta !^{\sigma}\langle\xi\rangle^{h-|\alpha|}, \quad h \in(0,1), \sigma \geq 1,
$$

with $c_{\alpha, \beta} \in L^{1}[0, T], \alpha, \beta \in Z_{+}^{n}$;

3) we give an energy estimate for the operator $L$ in Sobolev-Gevrey spaces of index $\sigma<1 / h$, with loss of derivatives.

A similar technique has already been used in [1], [2] to give results of $C^{\infty}$ and $G^{\sigma}$ well posedness for a Cauchy problem of the form (1.3), (1.4), (1.5) in the case of $\alpha \in C^{\infty}$ with zeroes of a finite order $k$, and under a Levi condition of the form (1.7) with $\gamma=1 / 2-1 / k$. In particular in [2] Gevrey well posedness for $\gamma<1 / 2-1 / k$ is proved.

\section{Gevrey Well Posedness for the Homogeneous Equation}

Let us consider the Cauchy problem (1.3) for the homogeneous operator $P$ given by (1.4) with $b \equiv c \equiv 0$. For the coefficients of

$$
P=D_{t}^{2}-\alpha(t) Q\left(t, x, D_{x}\right)
$$

we require assumptions (1.5), (1.6) to be satisfied.

We are going to find the sharp index $\sigma$ of Gevrey well posedness, depending on $N$ and $\chi$. More precisely, results of well posedness are going to be found in the Sobolev-Gevrey space $H^{s, \varepsilon, \sigma}$, where for $\varepsilon \geq 0, \sigma \geq 1$ we define

$$
H^{s, \varepsilon, \sigma}\left(\mathbf{R}^{n}\right)=\left\{u(x) ; e^{\varepsilon\left\langle D_{x}\right\rangle^{1 / \sigma}} u \in H^{s}\left(\mathbf{R}^{n}\right)\right\} ;
$$

in $H^{s, \varepsilon, \sigma}$ the norm is

$$
\|u\|_{s, \varepsilon, \sigma}=\left\|e^{\varepsilon\left\langle D_{x}\right\rangle^{1 / \sigma}} u\right\|_{s},
$$

and $\|u\|_{s}$ denotes the usual norm of $u$ in the Sobolev space $H^{s}$. Notice that

$$
H^{s, \varepsilon, \sigma} \subset G^{\sigma}, \quad \varepsilon>0 .
$$

Bounded pseudodifferential operators in these spaces are given by the classes $S^{m, \sigma}$ of Gevrey symbols. We remind that, for $m \in \mathbf{N}$ and $\sigma \geq 1, S^{m, \sigma}=S^{m, \sigma}\left(\mathbf{R}^{n} \times \mathbf{R}^{n}\right)$ denotes the space of all functions $a(x, \xi)$ satisfying

$$
\left|\partial_{\xi}^{\alpha} \partial_{x}^{\beta} a(x, \xi)\right| \leq C_{\alpha} M^{|\beta|} \beta !^{\sigma}\langle\xi\rangle^{m-|\alpha|}, \quad \alpha, \beta \in \mathbf{Z}_{+}^{n}, M, c_{\alpha}>0, x, \xi \in \mathbf{R}^{n},
$$

which is the limit space

$$
S^{m, \sigma}:=\lim _{\ell \rightarrow+\infty} S_{\ell}^{m, \sigma}, \quad S_{\ell}^{m, \sigma}:=\lim _{M \rightarrow+\infty} S_{\ell, M}^{m, \sigma}
$$


of the Banach spaces $S_{M, \ell}^{m, \sigma}$ of all symbols such that

$$
|a|_{m, \sigma, M, \ell}:=\sup _{|\alpha| \leq \ell, \beta \in \mathbf{Z}_{+}^{n}} \sup _{x, \xi}\left|\partial_{\xi}^{\alpha} \partial_{x}^{\beta} a(x, \xi)\right| M^{-|\beta|} \beta !^{-\sigma}\langle\xi\rangle^{-m+|\alpha|}<+\infty .
$$

The result of well posedness is presented in the following Theorem:

THEOREM 2.1. Consider the Cauchy problem (1.3) for the operator (2.1) under conditions (1.5), (1.6).

i) If $\chi<\frac{N}{N+2}$, then problem (1.3) is $G^{\sigma}$ well posed, $1 \leq \sigma<\frac{1}{1-\chi}$.

ii) If $\chi \geq \frac{N}{N+2}$, then problem (1.3) is $G^{\sigma}$ well posed, $1 \leq \sigma<1+\frac{N}{2}$.

Moreover, denoting by $\sigma_{0}$ the bound $1+N / 2$ or $1 /(1-\chi)$ according to the case we have the following: there exists a positive constant $\lambda$ such that for any $\mu \in \mathbf{R}$, $u_{0}, u_{1} \in H^{\mu, \lambda, \sigma}, f \in C\left([0, T] ; G^{\sigma}\right), 1 \leq \sigma<\sigma_{0}$, the unique solution $u(t, x)$ of $(1.3)$ satisfies the following energy estimate:

$$
\begin{aligned}
& \|u(t, \cdot)\|_{\mu, \lambda-\int_{0}^{t} \rho(\tau) d \tau, \sigma}^{2}+\left\|\partial_{t} u(t, \cdot)\right\|_{\mu, \lambda-\int_{0}^{t} \varphi(\tau) d \tau, \sigma}^{2} \\
& \quad \leq C_{\mu}\left(\left\|u_{0}\right\|_{\mu, \lambda, \sigma}^{2}+\left\|u_{1}\right\|_{\mu, \lambda, \sigma}^{2}+\int_{0}^{t}\|f(\tau, \cdot)\|_{\mu, \lambda-\int_{0}^{\tau} \varphi(s) d s, \sigma}^{2}\right)
\end{aligned}
$$

for every $u \in C\left([0, T] ; H^{\mu+1, \lambda, \sigma}\right) \cap C^{1}\left([0, T] ; H^{\mu, \lambda, \sigma}\right)$, and for a non negative function $\varphi \in L^{1}([0, T]), T \ll$.

Proof. The proof is divided into 3 steps, according to the scheme described in the introduction.

Step 1. The first step in the proof is to approximate the characteristic roots

$$
\pm \lambda(t, x, \xi)= \pm \sqrt{\alpha(t) Q(t, x, \xi)}
$$

of $P$ with positive and regular symbols $\pm \tilde{\lambda}(t, x, \xi)$ of order one, and then to factorize $P$ by means of the approximated characteristic roots.

Given a function

$$
\varrho \in C_{0}^{\infty}(\mathbf{R}), \quad 0 \leq \varrho \leq 1, \quad \int \varrho(\tau) d \tau=1,
$$

and extended the symbol $Q$ on $\mathbf{R}$ by considering

$$
Q(\tau, x, \xi)=Q(T, x, \xi) \text { for } \tau \geq T, \quad Q(\tau, x, \xi)=Q(0, x, \xi) \text { for } \tau \leq 0,
$$


Gevrey well posedness for a weakly hyperbolic equation in Gevrey classes

we define:

$$
\tilde{\lambda}(t, x, \xi)=\sqrt{\alpha(t)+\langle\xi\rangle^{-2 \chi}} \int \sqrt{Q(\tau, x, \xi)} \varrho(\langle\xi\rangle(t-\tau))\langle\xi\rangle d \tau .
$$

In order to factorize the operator $P$ by means of the regularized roots $\pm \tilde{\lambda}$ we need to consider the symbols $\partial_{t} \tilde{\lambda}$ and $\lambda-\tilde{\lambda}$.

We have

$$
\begin{aligned}
\partial_{t} \tilde{\lambda}(t, x, \xi)= & \frac{i \alpha^{\prime}(t)}{2 \sqrt{\alpha(t)+\langle\xi\rangle^{-2 \chi}}} \int \sqrt{Q(\tau, x, \xi)} \varrho(\langle\xi\rangle(t-\tau))\langle\xi\rangle d \tau \\
& +\sqrt{\alpha(t)+\langle\xi\rangle^{-2 \chi}} \int \sqrt{Q(\tau, x, \xi)} \varrho^{\prime}(\langle\xi\rangle(t-\tau))\langle\xi\rangle^{2} d \tau ;
\end{aligned}
$$

using the change of variables $\langle\xi\rangle(t-\tau)=s$ it is easy to find that

$$
S_{1}(t, x, \xi)=\int \sqrt{Q(\tau, x, \xi)} \varrho(\langle\xi\rangle(t-\tau))\langle\xi\rangle d \tau \in L^{1}\left([0, T] ; S^{1, \sigma}\right)
$$

using now $\int \varrho^{\prime}(s) d s=0$ we can write

$$
\begin{aligned}
& \int \sqrt{Q(\tau, x, \xi)} \varrho^{\prime}(\langle\xi\rangle(t-\tau))\langle\xi\rangle^{2} d \tau \\
& \quad=\int(\sqrt{Q(\tau, x, \xi)}-\sqrt{Q(t, x, \xi)}) \varrho^{\prime}(\langle\xi\rangle(t-\tau))\langle\xi\rangle^{2} d \tau,
\end{aligned}
$$

and then with the same change of variables and thanks to the second condition in (1.6) we obtain

$$
S_{2}(t, x, \xi)=\int \sqrt{Q(\tau, x, \xi)} \varrho^{\prime}(\langle\xi\rangle(t-\tau))\langle\xi\rangle^{2} d \tau \in L^{1}\left([0, T] ; S^{2-\chi, \sigma}\right) .
$$

Thus

$$
\left\{\begin{array}{l}
\partial_{t} \tilde{\lambda}(t, x, \xi)=\frac{i \alpha^{\prime}(t)}{2 \sqrt{\alpha(t)+\langle\xi\rangle^{-2 \chi}}} S_{1}(t, x, \xi)+\sqrt{\alpha(t)+\langle\xi\rangle^{-2 \chi}} S_{2}(t, x, \xi) \\
S_{1} \in L^{1}\left([0, T] ; S^{1, \sigma}\right), \quad S_{2} \in L^{1}\left([0, T] ; S^{2-\chi, \sigma}\right)
\end{array}\right.
$$

Moreover, looking at (2.3) and using $\int \varrho(s) d s=1$ we have 


$$
\begin{aligned}
\tilde{\lambda}(t, x, \xi)-\lambda(t, x, \xi) & \\
= & \sqrt{\alpha(t)+\langle\xi\rangle^{-2 \chi}} \int(\sqrt{Q(\tau, x, \xi)}-\sqrt{Q(t, x, \xi)}) \varrho(\langle\xi\rangle(t-\tau))\langle\xi\rangle d \tau \\
& +\left(\sqrt{\alpha(t)+\langle\xi\rangle^{-2 \chi}}-\sqrt{\alpha(t)}\right) \sqrt{Q(t, x, \xi)} .
\end{aligned}
$$

A simple computation and again the use of the second hypothesis in condition (1.6) makes clear that

$$
\int(\sqrt{Q(\tau, x, \xi)}-\sqrt{Q(t, x, \xi)}) \varrho(\langle\xi\rangle(t-\tau))\langle\xi\rangle d \tau \in L^{1}\left([0, T] ; S^{1-\chi, \sigma}\right)
$$

and also

$$
\left(\sqrt{\alpha(t)+\langle\xi\rangle^{-2 \chi}}-\sqrt{\alpha(t)}\right) \sqrt{Q(t, x, \xi)} \in L^{1}\left([0, T] ; S^{1-\chi, \sigma}\right) .
$$

Thus

$$
\tilde{\lambda}-\lambda \in L^{1}\left([0, T] ; S^{1-\chi, \sigma}\right) .
$$

By (2.7) we have also

$$
\left\{\begin{array}{l}
{\left[\tilde{\lambda}\left(t, x, D_{x}\right)\right]^{2}-\left[\lambda\left(t, x, D_{x}\right)\right]^{2}=\sqrt{\alpha(t)+\left\langle D_{x}\right\rangle^{-2 \chi}} S_{3}\left(t, x, D_{x}\right),} \\
S_{3}(t, x, \xi) \in L^{1}\left([0, T] ; S^{2-\chi, \sigma}\right) .
\end{array}\right.
$$

Now from (2.6), (2.8) we come to the factorization

$$
\begin{aligned}
D_{t}^{2}-\alpha(t) Q\left(t, x, D_{x}\right) \\
=\left(D_{t}-\tilde{\lambda}\left(t, x, D_{x}\right)\right)\left(D_{t}+\tilde{\lambda}\left(t, x, D_{x}\right)\right)+\frac{i \alpha^{\prime}(t)}{2 \sqrt{\alpha(t)+\left\langle D_{x}\right\rangle^{-2 \chi}}} S_{1}\left(t, x, D_{x}\right) \\
\quad+\frac{\alpha(t)}{4 Q\left(t, x, D_{x}\right)} \sum_{j=1}^{n} \partial_{\xi_{j}} Q\left(t, x, D_{x}\right) D_{x_{j}} Q\left(t, x, D_{x}\right) \\
\quad+\sqrt{\alpha(t)+\left\langle D_{x}\right\rangle^{-2 \chi}} S_{4}\left(t, x, D_{x}\right)+R\left(t, x, D_{x}\right),
\end{aligned}
$$

with

$$
\left\{\begin{array}{l}
S_{1}(t, x, \xi) \in L^{1}\left([0, T] ; S^{1, \sigma}\right) \\
S_{4}(t, x, \xi)=S_{2}(t, x, \xi)+S_{3}(t, x, \xi) \in L^{1}\left([0, T] ; S^{2-\chi, \sigma}\right) \\
R(t, x, \xi) \in C\left([0, T] ; S^{0, \sigma}\right)
\end{array}\right.
$$


Step 2. The second step in the proof is to reduce the equation $P u=f$ for the scalar operator $P$ in (2.1) to an equivalent first order system by using factorization (2.9). We define

$$
\omega(t, \xi)=\sqrt{\alpha(t)+\langle\xi\rangle^{-2 \chi}}\langle\xi\rangle
$$

and

$$
\left\{\begin{array}{l}
v_{0}=\omega\left(t, D_{x}\right) u \\
v_{1}=\left(D_{t}+\tilde{\lambda}\left(t, x, D_{x}\right)\right) u
\end{array}\right.
$$

Notice that $\omega \in C\left([0, T] ; S^{1}\right), \omega^{-1} \in C\left([0, T] ; S^{0}\right)$. We have

$$
\begin{aligned}
\left(D_{t}+\tilde{\lambda}\right) v_{0}= & \sqrt{\alpha(t)+\left\langle D_{x}\right\rangle^{-2 \chi}}\left\langle D_{x}\right\rangle v_{1} \\
& +\frac{i \alpha^{\prime}(t)}{2\left(\alpha(t)+\left\langle D_{x}\right\rangle^{-2 \chi}\right)} v_{0}+[\tilde{\lambda}, \omega] \omega^{-1} v_{0},
\end{aligned}
$$

where the operator $[\tilde{\lambda}, \omega] \omega^{-1}$ is of order 0 , and

$$
\begin{aligned}
\left(D_{t}-\tilde{\lambda}\right) v_{1} & \\
& =\left(\frac{\alpha^{\prime}(t)}{\alpha(t)+\left\langle D_{x}\right\rangle^{-2 \chi}} T_{1}+\frac{\alpha(t)}{\sqrt{\alpha(t)+\left\langle D_{x}\right\rangle^{-2 \chi}}} T_{2}+T_{3}+T_{4}\right) v_{0},
\end{aligned}
$$

$T_{j}=T_{j}\left(t, x, D_{x}\right), \quad$ with $\quad T_{3}(t, x, \xi) \in C\left([0, T] ; S^{1-\chi, \sigma}\right), \quad T_{1}(t, x, \xi), T_{2}(t, x, \xi)$, $T_{4}(t, x, \xi) \in C\left([0, T] ; S^{0, \sigma}\right)$.

Then, from (2.11), (2.13), (2.14), problem (1.3) is equivalent to the Cauchy problem

$$
\left\{\begin{array}{l}
L_{1} V=0, \\
V(0, x)=V_{0},
\end{array}\right.
$$

for the first order system

$$
\begin{aligned}
L_{1}= & \partial_{t}+i\left(\begin{array}{cc}
\tilde{\lambda}\left(t, x, D_{x}\right) & -\omega\left(t, D_{x}\right) \\
0 & -\tilde{\lambda}\left(t, x, D_{x}\right)
\end{array}\right) \\
& +\frac{\alpha^{\prime}(t)}{\alpha(t)+\left\langle D_{x}\right\rangle^{-2 \chi}} A_{1}\left(t, x, D_{x}\right)+C_{1}\left(t, x, D_{x}\right) \\
& +\frac{\alpha(t)}{\sqrt{\alpha(t)+\left\langle D_{x}\right\rangle^{-2 \chi}}} D_{1}\left(t, x, D_{x}\right)+E_{1}\left(t, x, D_{x}\right),
\end{aligned}
$$


where $A_{1}(t, x, \xi), C_{1}(t, x, \xi), D_{1}(t, x, \xi), E_{1}(t, x, \xi)$ are $2 \times 2$ matrices such that

$$
\left\{\begin{array}{l}
A_{1}, D_{1}, E_{1} \in C\left([0, T] ; S^{0, \sigma}\right), \\
C_{1} \in C\left([0, T] ; S^{1-\chi, \sigma}\right)
\end{array}\right.
$$

To diagonalize the matrix

$$
\left(\begin{array}{cc}
\tilde{\lambda}(t, x, \xi) & -\omega(t, \xi) \\
0 & -\tilde{\lambda}(t, x, \xi)
\end{array}\right)
$$

we use

$$
M(t, x, \xi)=\left(\begin{array}{cc}
1 & \frac{\langle\xi\rangle}{2 S_{1}(t, x, \xi)} \\
0 & 1
\end{array}\right),
$$

$S_{1}$ in (2.4), which is elliptic of order zero. Thus problem (2.15) is equivalent to

$$
\left\{\begin{array}{l}
L U=0 \\
U(0, x)=U_{0}
\end{array}\right.
$$

where $U=M\left(t, x, D_{x}\right) V$ and

$$
\begin{gathered}
L=\partial_{t}+i \Lambda\left(t, x, D_{x}\right)+\frac{\alpha^{\prime}(t)}{\alpha(t)+\left\langle D_{x}\right\rangle^{-2 \chi}} A\left(t, x, D_{x}\right)+C\left(t, x, D_{x}\right) \\
+\frac{\alpha(t)}{\sqrt{\alpha(t)+\left\langle D_{x}\right\rangle^{-2 \chi}}} D\left(t, x, D_{x}\right)+E\left(t, x, D_{x}\right), \\
\Lambda\left(t, x, D_{x}\right)=\left(\begin{array}{cc}
\tilde{\lambda}\left(t, x, D_{x}\right) & 0 \\
0 & -\tilde{\lambda}\left(t, x, D_{x}\right)
\end{array}\right),
\end{gathered}
$$

with new $2 \times 2$ matrices

$$
\left\{\begin{array}{l}
A(t, x, \xi), D(t, x, \xi), E(t, x, \xi) \in C\left([0, T] ; S^{0, \sigma}\right), \\
C(t, x, \xi) \in C\left([0, T] ; S^{1-\chi, \sigma}\right) .
\end{array}\right.
$$

Looking now at the terms in (2.17) we have that

$$
\frac{\alpha(t)}{\sqrt{\alpha(t)+\langle\xi\rangle^{-2 \chi}}} D(t, x, \xi) \leq \sqrt{\alpha(t)+\langle\xi\rangle^{-2 \chi}} D(t, x, \xi) \in C\left([0, T] ; S^{0, \sigma}\right),
$$

while

$$
\begin{aligned}
\tilde{A}(t, x, \xi) & =\frac{\alpha^{\prime}(t)}{\alpha(t)+\langle\xi\rangle^{-2 \chi}} A(t, x, \xi) \\
& =\frac{\alpha^{\prime}(t)}{\left(\alpha(t)+\langle\xi\rangle^{-2 \chi}\right)^{1-1 / N}} \cdot \frac{A(t, x, \xi)}{\left(\alpha(t)+\langle\xi\rangle^{-2 \chi}\right)^{1 / N}}
\end{aligned}
$$


is such that for all $\beta, \gamma \in Z_{+}^{n}$ it is

$$
\left|\partial_{\xi}^{\beta} \partial_{x}^{\gamma} \tilde{A}(t, x, \xi)\right| \leq c_{\beta, \gamma} \frac{\alpha^{\prime}(t)}{\alpha(t)+\langle\xi\rangle^{-2 \chi}}\langle\xi\rangle^{-|\beta|} ;
$$

the symbol $\tilde{A} \in L^{1}\left([0, T] ; S^{2 \chi / N}\right)$, since if $\alpha \in C^{N}$, then $\alpha^{1 / N}$ is an absolutely continuous function (see Lemma 1 in [5]).

Here we need to distinguish two cases because the orders $2 \chi / N$ and $1-\chi$ fight one against the other. We have

$$
2 \chi / N=1-\chi \text { for } \chi=N /(2+N) .
$$

Thus:

i) if $\chi<\frac{N}{N+2}$, then the $C^{\chi}$ behavior dominates, and the Cauchy problem (2.16) for the operator (2.17) can be written in the form

$$
\left\{\begin{array}{l}
\left(\partial_{t}+i \Lambda\left(t, x, D_{x}\right)+R\left(t, x, D_{x}\right)\right) U(t, x)=0, \\
U(0, x)=U_{0}
\end{array}\right.
$$

with

$$
R(t, x, \xi) \in L^{1}\left([0, T] ; S^{1-\chi, \sigma}\right) .
$$

It is well known that to have existence and uniqueness of the solution of such a Cauchy problem one has to require that the Gevrey index is

$$
\sigma<\frac{1}{1-\chi}:=\sigma_{0}
$$

ii) if $\chi \geq \frac{N}{N+2}$, then the $C^{N}$ behavior dominates, and the Cauchy problem (2.16) for the operator (2.17) can be written again in the form (2.20) but this time with

$$
R(t, x, \xi) \in L^{1}\left([0, T] ; S^{2 /(N+2), \sigma}\right) .
$$

In order to have existence and uniqueness of the solution we need to ask

$$
\sigma<\left(\frac{2}{N+2}\right)^{-1}=1+\frac{N}{2}:=\sigma_{0}
$$

Step 3. Starting from now, we will denote by $\sigma_{0}$ the bound $1+N / 2$ or $1 /(1-\chi)$ according to the case we are considering. We give hereafter the energy estimate for the operator 


$$
L=\partial_{t}+i \Lambda\left(t, x, D_{x}\right)+R\left(t, x, D_{x}\right)
$$

$\Lambda$ in (2.18), with

$$
R(t, x, \xi) \in L^{1}\left([0, T] ; S^{1 / \sigma_{0}, \sigma}\right),
$$

by proving the following:

ENERGY ESTIMATE. There exists a positive constant $\lambda$ such that for any $\mu \in \mathbf{R}$, $U \in H^{\mu, \lambda, \sigma}, \quad F \in C\left([0, T] ; G^{\sigma}\right), \quad 1 \leq \sigma<\sigma_{0}$, the unique solution $U(t, x)$ of the Cauchy problem

$$
\left\{\begin{array}{l}
L U=0 \\
U(0)=U_{0}
\end{array}\right.
$$

$L$ in (2.21), satisfies the estimate:

$$
\|U(t, \cdot)\|_{\mu, \lambda-\int_{0}^{t} \varphi(\tau) d \tau, \sigma}^{2} \leq C_{\mu}\left(\left\|U_{0}\right\|_{\mu, \lambda, \sigma}^{2}+\int_{0}^{t}\|F(\tau, \cdot)\|_{\mu, \lambda-\int_{0}^{\tau} \varphi(s) d s, \sigma}^{2}\right),
$$

for every $U \in C\left([0, T] ; H^{\mu+1, \lambda, \sigma}\right) \cap C^{1}\left([0, T] ; H^{\mu, \lambda, \sigma}\right)$, and for a non negative function $\varphi \in L^{1}([0, T]), T<$.

Proof of the energy estimate. We give the energy estimate only for $\mu=0$, because if $\mu \neq 0$ we have

$$
\left\langle D_{x}\right\rangle^{-\mu} L\left\langle D_{x}\right\rangle^{\mu}=L+R_{\mu},
$$

$R_{\mu}$ of order zero, so we can always reduce to the case $\mu=0$ without any loss of generality.

From (2.17) and (2.19), it is clear the existence of a function $\varphi(t) \geq 0$, $\varphi \in L^{1}([0, T])$, such that

$$
\left|\partial_{\xi}^{\alpha} \partial_{x}^{\beta} R(t, x, \xi)\right| \leq c_{\alpha, \beta} \varphi(t)\langle\xi\rangle^{1 / \sigma_{0}-|\alpha|}, \quad \alpha, \beta \in \mathbf{Z}_{+}^{n}, x, \xi \in \mathbf{R}^{n} .
$$

Let us define the operator $w\left(t, D_{x}\right)$ with symbol

$$
w(t, \xi)=e^{\left(\lambda-\int_{0}^{t} \varphi(\tau) d \tau\right)\langle\xi\rangle^{1 / \sigma_{0}}}
$$

$\lambda>0$ to be choosen, and consider the operator

$$
L_{w}=w L w^{-1} .
$$

The Cauchy problem $L U=0, U(0)=U_{0}$ is equivalent to 
Gevrey well posedness for a weakly hyperbolic equation in Gevrey classes

$$
\left\{\begin{array}{l}
L_{w} Z=0 \\
Z(0)=Z_{0},
\end{array}\right.
$$

with

$$
L_{w}=\partial_{t}+i\left(\begin{array}{cc}
\tilde{\lambda} & 0 \\
0 & -\tilde{\lambda}
\end{array}\right)+\varphi(t)\left\langle D_{x}\right\rangle+R+\text { lower order terms }
$$

on condition that

$$
\int_{0}^{T} \varphi(t) d t \leq \lambda, \quad \lambda \leq \lambda_{0}
$$

(see the composition theorems in [7]), and with

$$
Z(t, x)=e^{\left(\lambda-\int_{0}^{t} \varphi(\tau) d \tau\right)\langle\xi\rangle^{1 / \sigma_{0}}} U(t, x) .
$$

The operator $\varphi(t)\left\langle D_{x}\right\rangle+R$ is positively defined because

$$
|\langle R Z, Z\rangle| \leq\left\langle\varphi(t)\left\langle D_{x}\right\rangle^{1 / \sigma_{0}} Z, Z\right\rangle,
$$

where $\langle\cdot, \cdot\rangle$ denotes the scalar product in $H^{0}\left(\mathbf{R}^{n}\right)=L^{2}\left(\mathbf{R}^{n}\right)$.

Consider now

$$
\frac{d}{d t}\|Z(t)\|_{0}^{2}=2 \Re\left\langle Z^{\prime}(t), Z(t)\right\rangle .
$$

From (2.25), (2.27), we have

$$
\frac{d}{d t}\|Z(t)\|_{0}^{2} \leq C\left(\|Z(t)\|_{0}^{2}+\left\|L_{w} Z(t)\right\|_{0}^{2}\right) .
$$

Thus, by Gronwall's inequality we find

$$
\|Z(t)\|_{0}^{2} \leq C_{0}\left(\left\|Z_{0}\right\|_{0}^{2}+\int_{0}^{t}\left\|L_{w} Z(\tau)\right\|_{0}^{2} d \tau\right) .
$$

Now to obtain (2.22) one only has to remember the definition of $Z$ in (2.26). Estimate (2.2) follows then immediately from (2.22), using (2.12). Notice that transformation (2.23) carries the loss of $\int_{0}^{t} \varphi(\tau) d \tau$ derivatives, with $T \ll$. The maximum loss is so

$$
\int_{0}^{T} \varphi(\tau) d \tau<+\infty
$$

Theorem (2.1) is completely proved. 


\section{Gevrey Well Posedness under Levi Conditions}

In this last section of the paper we investigate the behavior of the lower order terms $b\left(t, x, D_{x}\right), c(t, x)$ in equation (1.4) with respect to the results of Gevrey well posedness of Theorem 2.1.

We consider here the Cauchy problem (1.3) in the general case of $P$ given by (1.4). We assume the coefficients of (1.4) to satisfy (1.5), (1.6), and we add for the lower order terms condition (1.7).

Then we have the following result of well posedness under Levi conditions:

THEOREM 3.1. Consider the Cauchy problem (1.3) for $P$ in (1.4) and under conditions (1.5), (1.6), (1.7).

i) If $\chi<\frac{N}{N+2}$ and $\gamma \geq 1-\frac{1}{2 \chi}$, then problem (1.3) is $G^{\sigma}$ well posed for every $1 \leq \sigma<\frac{1}{1-\chi}$.

ii) If $\chi \geq \frac{N}{N+2}$ and $\gamma \geq \frac{1}{2}-\frac{1}{N}$, then problem (1.3) is $G^{\sigma}$ well posed for every $1 \leq \sigma<1+\frac{N}{2}$.

Moreover, the energy estimate (2.2) for the solution $u(t, x)$ of (1.3) remains valid.

Proof. The proof will follow the one of Theorem 2.1 with the addition of the lower order terms $b, c$ at each step.

Like in Step 1, we approximate the characteristic roots by defining $\tilde{\lambda}$ as in (2.3), and we obtain from (1.4), (2.9) the factorization

$$
\begin{aligned}
& P\left(t, x, D_{x}\right) \\
& =\left(D_{t}-\tilde{\lambda}\left(t, x, D_{x}\right)\right)\left(D_{t}+\tilde{\lambda}\left(t, x, D_{x}\right)\right)+\frac{i \alpha^{\prime}(t)}{2 \sqrt{\alpha(t)+\left\langle D_{x}\right\rangle^{-2 \chi}}} S_{1}\left(t, x, D_{x}\right) \\
& +\frac{\alpha(t)}{4 Q\left(t, x, D_{x}\right)} \sum_{j=1}^{n} \partial_{\xi_{j}} Q\left(t, x, D_{x}\right) D_{x_{j}} Q\left(t, x, D_{x}\right) \\
& +\sqrt{\alpha(t)+\left\langle D_{x}\right\rangle^{-2 \chi}} S_{4}\left(t, x, D_{x}\right)+b\left(t, x, D_{x}\right)+c(t, x)+R\left(t, x, D_{x}\right),
\end{aligned}
$$
$C\left([0, T] ; S^{0, \sigma}\right)$.

As in Step 2, we perfom the reduction (2.11), (2.12). We obtain (2.13) and 
Gevrey well posedness for a weakly hyperbolic equation in Gevrey classes

$$
\begin{aligned}
\left(D_{t}-\tilde{\lambda}\right) v_{1}= & \left(\frac{\alpha^{\prime}(t)}{\alpha(t)+\left\langle D_{x}\right\rangle^{-2 \chi}} T_{1}+\frac{\alpha(t)}{\sqrt{\alpha(t)+\left\langle D_{x}\right\rangle^{-2 \chi}}} T_{2}+T_{3}\right. \\
& \left.-\frac{b\left(t, x, D_{x}\right)}{\sqrt{\alpha(t)+\left\langle D_{x}\right\rangle^{-2 \chi}}\left\langle D_{x}\right\rangle}+T_{4}\right) v_{0},
\end{aligned}
$$

$T_{j}=T_{j}\left(t, x, D_{x}\right), \quad$ with $\quad T_{3}(t, x, \xi) \in C\left([0, T] ; S^{1-\chi, \sigma}\right), \quad T_{1}(t, x, \xi), T_{2}(t, x, \xi)$, $T_{4}(t, x, \xi) \in C\left([0, T] ; S^{0, \sigma}\right)$.

Then, from (2.11), (2.13), (3.2) and after the diagonalization of the principal part of the system performed in the previous section, the Cauchy problem (1.3) is equivalent to problem

$$
\left\{\begin{array}{l}
L U=0 \\
U(0, x)=U_{0}
\end{array}\right.
$$

where

$$
\begin{aligned}
L= & \partial_{t}+i \Lambda\left(t, x, D_{x}\right)+\frac{\alpha^{\prime}(t)}{\alpha(t)+\left\langle D_{x}\right\rangle^{-2 \chi}} A\left(t, x, D_{x}\right) \\
& +\frac{b\left(t, x, D_{x}\right)}{\sqrt{\alpha(t)+\left\langle D_{x}\right\rangle^{-2 \chi}}\left\langle D_{x}\right\rangle} B\left(t, x, D_{x}\right)+C\left(t, x, D_{x}\right) \\
& +\frac{\alpha(t)}{\sqrt{\alpha(t)+\left\langle D_{x}\right\rangle^{-2 \chi}}} D\left(t, x, D_{x}\right)+E\left(t, x, D_{x}\right)
\end{aligned}
$$

$\Lambda$ in (2.18), with

$$
\left\{\begin{array}{l}
A(t, x, \xi), B\left(t, x, D_{x}\right), D(t, x, \xi), E(t, x, \xi) \in C\left([0, T] ; S^{0, \sigma}\right) \\
C(t, x, \xi) \in C\left([0, T] ; S^{1-\chi, \sigma}\right)
\end{array}\right.
$$

To deal with the new term $\frac{b\left(t, x, D_{x}\right)}{\sqrt{\alpha(t)+\left\langle D_{x}\right\rangle^{-2 x}}\left\langle D_{x}\right\rangle} B\left(t, x, D_{x}\right)$ we use the Levi condition (1.7) to write

$$
\frac{b(t, x, \xi)}{\sqrt{\alpha(t)+\langle\xi\rangle^{-2 \chi}}\langle\xi\rangle} B(t, x, \xi)=\frac{b(t, x, \xi)}{\left(\alpha(t)+\langle\xi\rangle^{-2 \chi}\right)^{\gamma}\langle\xi\rangle} \cdot \frac{B(t, x, \xi)}{\left(\alpha(t)+\langle\xi\rangle^{-2 \chi}\right)^{1 / 2-\gamma}},
$$

where

$$
\frac{b(t, x, \xi)}{\left(\alpha(t)+\langle\xi\rangle^{-2 \chi}\right)^{\gamma}\langle\xi\rangle} \in C\left([0, T] ; S^{0, \sigma}\right)
$$


and

$$
\frac{B(t, x, \xi)}{\left(\alpha(t)+\langle\xi\rangle^{-2 \chi}\right)^{1 / 2-\gamma}} \in L^{1}\left([0, T] ; S^{2 \chi(1 / 2-\gamma)}\right) .
$$

Thus, according to Theorem 2.1 we distinguish two cases.

i) If $\chi<\frac{N}{N+2}$, we ask $2 \chi(1 / 2-\gamma) \leq 1-\chi$. We obtain that if

$$
\gamma \geq 1-\frac{1}{2 \chi}=\gamma_{0}
$$

then the Cauchy problem (3.3) for the operator (3.4) is of the form (2.20), with

$$
R(t, x, \xi) \in L^{1}\left([0, T] ; S^{1-\chi, \sigma}\right) .
$$

Gevrey well posedness holds so for

$$
\sigma<\frac{1}{1-\chi}=\sigma_{0} .
$$

ii) If $\chi \geq \frac{N}{N+2}$, we require $2 \chi(1 / 2-\gamma) \leq 2 \chi / N$. We obtain that if

$$
\gamma \geq \frac{1}{2}-\frac{1}{N}=\gamma_{0}
$$

the Cauchy problem (3.3) for the operator (3.4) is of the form (2.20), with

$$
R(t, x, \xi) \in L^{1}\left([0, T] ; S^{2 /(N+2), \sigma}\right) .
$$

Gevrey well posedness holds for

$$
\sigma<1+\frac{N}{2}=\sigma_{0}
$$

The energy estimate can be now derived from Step 3 of Theorem 2.1 exactly in the same way. Theorem 3.1 is proved.

REMARK 3.2. We finally notice that:

- the choice of $\gamma$ depends only on the dominant between $N$ and $\chi$;

- if $\gamma<\gamma_{0}$ (according to the case), then (3.3) can be written again in the form (2.20), but with a remainder

$$
R(t, x, \xi) \in L^{1}\left([0, T] ; S^{\gamma_{0}, \sigma}\right),
$$


and the Cauchy problem (1.3) is well posed only for

$$
\sigma<\frac{1}{\gamma_{0}}<\sigma_{0} .
$$

In such a case the lower order term $b$ destroys the well posedness results of Theorem 2.1.

\title{
References
}

[1] Ascanelli, A.; Cicognani, M.: Energy estimate and Fundamental Solution for Degenerate Hyperbolic Cauchy problems. J. Differential Equations 217 (2005), n. 2, 305-340.

[2] Ascanelli, A.; Cicognani, M.: Well posedness of the Cauchy problem for some degenerate hyperbolic operators. Pseudo-Differential Operators and Related Topics Series: Operator Theory: Advances and Applications, Vol. 164, Editors: P. Boggiatto, L. Rodino, J. Toft, M.-W. Wong, Birkhuser Verlag Basel/Switzerland (2006), 23-41.

[ 3 ] Bronšteïn, M. D.: The Cauchy problem for hyperbolic operators with characteristics of variable multiplicity. Trudy Moskov. Mat. Obshch. 41 (1980), 83-99.

[4] Colombini, F.; De Giorgi, E.; Spagnolo, S.: Sur les équations hyperboliques avec des coefficients qui ne dépendent que du temps. Ann. Sc. Norm. Sup. Pisa 6 (1979), 511-559.

[5] Colombini, F.; Jannelli, E.; Spagnolo, S.: Well-posedness in Gevrey classes of the Cauchy problem for a non strictly hyperbolic equation with coefficients depending on time. Ann. Scuola Norm. Sup. Pisa Cl. Sci. 10 (1983), 291-312.

[6] Colombini, F.; Spagnolo, S.: An Example of a Weakly Hyperbolic Cauchy Problem not wellposed in $C^{\infty}$. Acta Math. 148 (1982), 243-253.

[7] Kajitani, K.: Cauchy problem for nonstrictly hyperbolic systems in Gevrey classes. J. Math. Kyoto Univ. 23-3 (1983), 599-616.

[8] Kajitani, K.: Local solution of Cauchy problem for nonlinear hyperbolic systems in Gevrey classes. Hokkaido Math. J. 12 (1983), 434-460.

[9] Mizohata, S.: On the Cauchy problem. Notes and Reports in Mathematics in Science and Engineering, 3. Academic Press, Inc., Orlando, FL; Science Press, Beijing, 1985.

[10] Nishitani, T.: The Cauchy problem for weakly hyperbolic equations of second order. Comm. Partial Differential Equations 5 (1980), 1273-1296.

[11] Nishitani, T.: Sur les équations hyperboliques a coefficients höld ériens en $t$ et de classe de Gevrey en $x$. Bull. Sc. Math. 107 (1983), 113-138.

[12] Nishitani, T.: On the Cauchy problem for $D_{t}^{2}-D_{x} a(t, x) D_{x}$. Preprint (2004).

\author{
Alessia Ascanelli \\ Dipartimento di Matematica \\ Università di Ferrara, Via Machiavelli 35 \\ 44100 Ferrara, Italy \\ E-mail address: alessia.ascanelli@unife.it
}

